\title{
Editorial
}

\section{THE FREE PUBLISHING PLATFORM IN GERONTOLOGY: INTRODUCING Ageing and Longevity (AEL)}

It is a great pleasure to announce that the new peer reviewed open an access Ukrainian journal of gerontology and geriatrics Ageing and Longevity is now operational; here we introduce you to the first issue which we hope you may find of interest. The journal is ready to receive submission and welcomes all members of gerontological and geriatric community in the Ukraine and around the world to submit and to publish with us.

Ageing and Longevity provides an accessible and multi-disciplinary platform for state of the art research in all aspects of theoretical and translational gerontology, in social gerontology and in clinical studies. Why do we move into a new platform and into open access? The publishing is changing very much as our whole world is going through the transition into the unknown. Our vision is to make Ageing and Longevity a home for all gerontologists regardless of their background and to provide a forum for free and world-wide exchange of ideas and knowledge. The journal will be published in English only; again this is fundamental for making it being read and being influential. English is the language of science and nothing can change it in a foreseeable future except the major cataclysm. At the same time we keep the journal free of charge, free for authors and for readers. For how long we will be able to maintain this seemingly unviable and idealistic model? Remains the question which we cannot answer in the current circumstances, yet we shall do our best. We rely on our authorship very much, we need a content of the highest quality and we need our authors to be our reviewers because the peer review is the only quality control.

The history of Ukrainian gerontology is unique in many senses. Father of modern gerontology and a Noble prize winner Élie Mechnikov started scientific career at the beginning of 
the $X X^{\text {th }}$ century in Odessa. In 1920s and 1930s, Universities of Kharkiv and Kyiv were leaders in research into ageing and longevity. In 1923, Professor Alexander Nagornyi (Kharkiv) published the book "Life, Old Age and Death". In 1926, Professor of Kyiv University Ivan Schmalgausen wrote a treatise on "Problems of Death and Immortality". Since 1930s, Kharkiv School of Gerontology became active in studying biochemistry of ageing. In 1940, professors Alexander Nagornyi and Ivan Bulankin published the book "Problem of ageing and longevity". In 1937, the first complex study of longevity, in Abkhazia was conducted by Ukrainian researchers, and in 1938 results of these studies were presented at the conference in Kyiv. After the Second World War researchers from Kharkiv University Group continued their studies of mechanisms of ageing, and began actively exploring experimental paradigms for life expansion; in particular Vladimir Nikitin was one of the very first to propose restrictive diet. In 1958, the Kiev Institute of Gerontology was established. Major areas of research in the Institute include experimental gerontology (mechanisms of ageing and longevity, means and ways of life extension), physiology and pathophysiology of human ageing (including study of population longevity, nonagenarians and centenarians), social gerontology and gerohygiene. The Institute participated in numerous international studies and organized hundreds of international and national scientific forums.

In 1962, the Scientific-medical Society of Gerontologists and Geriatricians of the Ukraine was established and it became a part of the International Association of Gerontology and Geriatrics. In 1972, the IXth World Congress of the International Association of Gerontology took place in Kiev attended by 3500 participants from all over the world. Dozens of yearbooks and monographs on various aspects of gerontology and geriatrics and thousands of papers were published in these years. In 1997, the Society and the Institute established the quarterly journal "Problems of Ageing and Longevity", which at those days was published in Russian.

Times are changing. The mankind is getting older, and hence humanitarian, socioeconomic and medical aspects of ageing and longevity are becoming urgent. Among challenges growing numbers of elderly and old people burdened with multiple chronic diseases and incapacities; increased need of the elderly population in various types of assistance and care; economic and infrastructural challenges to societies; notwithstanding more and more elderly stay in good health and are capable for productive and successful ageing and healthy longevity.

We live in the time of pandemic of COVID-19; something which has not happened since 1918. The pandemic raised once again the question on age-related peculiarities of diseases and mortality in old age. High incidence of the disease and its complications, high mortality-rate 
among elderly patients - all are consequences of many factors, like metabolic and functional changes in various systems of an ageing organism (immune, pulmonary, cardiovascular, endocrine etc.), chronic diseases burden (age-related immunodeficiency, pulmonary obstructive disease, bronchial asthma, diabetes, cardiovascular diseases etc.).

Recent discoveries in biomedical research raised hopes for slowing-down the ageing and development of strategies to prevent age-related pathology, with early diagnosis and treatment of some debilitating chronic diseases of old age, for achieving longer and healthier life. We hope that Ageing and Longevity will contribute to these advances.

\section{Vladislav Bezrukov \\ Alexei Verkhratsky \\ Editors in Chief}

\section{Anna Shershanova \\ Receiving editor}

Author Contributions: All authors participated equally in writing this commentary.

Conflicts of Interest: The authors declare no conflict of interest.

Information about Authors:

Vladislav Bezrukov - MD, DSc (Medicine), Prof., Full Member (Academician) of the NAMS of Ukraine, Director of the State Institution “D. F. Chebotarev Institute of Gerontology NAMS of Ukraine”, Head of the Laboratory of Physiology; https://orcid.org/0000-0002-3913-3818

Alexei Verkhratsky - MD, PhD, DSc, MAE, ML, MPAN, MSASA, Prof., Faculty of Biology, Medicine and Health, The University of Manchester, Manchester, M13 9PT, UK, Achucarro Center for Neuroscience IKERBASQUE, 48011 Bilbao, Spain ; https://orcid.org/0000-0003-2592-9898

Anna Shershanova - Senior Researcher, Receiving Editor of Journal "Ageing and Longevity" of the State Institution “D. F. Chebotarev Institute of Gerontology NAMS of Ukraine” 